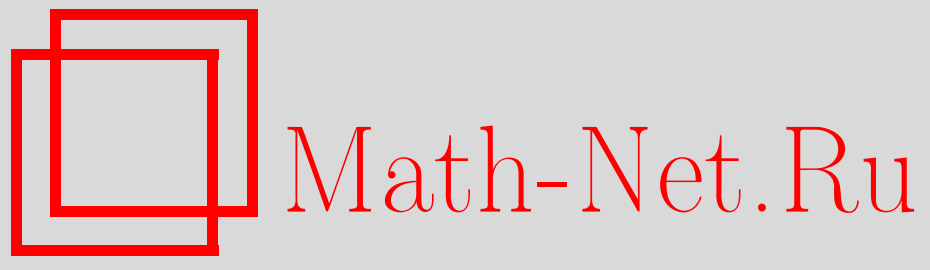

С. Я. Махно, Сходимость решений одномерных стохастических уравнений, Теория вероятн. и ее примен., 1999, том 44, выпуск $3,555-572$

DOI: https://doi.org/10.4213/tvp803

Использование Общероссийского математического портала Math-Net.Ru подразумевает, что вы прочитали и согласны с пользовательским соглашением http://www . mathnet.ru/rus/agreement

Параметры загрузки:

IP : 3.80 .181 .102

26 апреля 2023 г., $17: 56: 18$

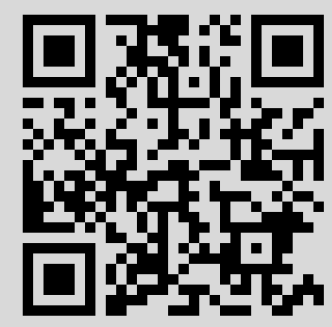




\title{
СХОДИМОСТЬ РЕШЕНИЙ ОДНОМЕРНЫХ СТОХАСТИЧЕСКИХ УРАВНЕНИЙ
}

\begin{abstract}
Рассматриваются одномерные стохастические уравнения, коэффициенты которых зависят от малого параметра нерегулярным образом. Получены необходимые и достаточные условия слабой сходимости их решений к решению стохастического уравнения в терминах коэффициентов. Приведены примеры.
\end{abstract}

Ключевые слова и фразы: стохастические уравнения, предельные теоремы, необходимые условия сходимости, достаточные условия сходимости.

0. Введение. В работе рассматриватотся решения одномерных однородных стохастических уравнений, зависящих от малого параметра $\varepsilon$, и изучается их слабая сходимость при $\varepsilon \rightarrow 0$. При этом не предполагается ни равномерная ограниченность по параметру коэффициента сноса, ни существование пределов у коэффищиентов уравнения при $\varepsilon \rightarrow 0$. Предельный процесс также будет решением стохастического уравнения. Получены необходимые и достаточные условия слабой сходимости решений стохастических уравнений в терминах их коэффициентов.

Близкая по постановке проблема рассматривалась в работе [4]. Однако там исследовалась слабая сходимость преобразований от решений уравнений, уничтожающих снос.

Работа построена по следующему плану. В этом пункте вводятся предположения и формулируется теорема, содержашая основные результаты статьи. Доказательство теоремы проводится по частям в пाг. 1 и 2. Вспомогательные результаты доказываются в п. 3, в п. 4 рассмотрены конкретные классы случайных процессов и исследуется их поведение, основываясь на полученных результатах.

Обозначим $\left(\Omega, \mathscr{F}, \mathscr{F}_{t}, \mathrm{P}\right)$ основное вероятностное пространство с потоком $\sigma$-алгебр $\mathscr{F}_{t}, t \geqslant 0, E_{1}$ - одномерное евклидово пространство,

\footnotetext{
${ }^{*}$ Институт прикладной математики и механики НАН Украины, ул. Р. Люксембург,
} 74, 340114 Донещк, Украина; e-mail: makhno@iamm.ac.donetsk.ua

1) Работа выполнена при поддержке гранта INTAS 94-0378. 
Е-символ математического ожидания. Рассматриваемые решения стохастических уравнений будут марковскими процессами. Поэтому будет использоваться обычная символика теории марковских процессов. Производная функции $f(x)$ будет обозначаться $\dot{f}(x)$. Через $C, K, k, N$ будут обозначаться различные постоянные, с индексами, если это необходимо, $\chi(A)$ - индикатор множества $A$.

Пусть

$$
\begin{aligned}
\xi_{\varepsilon}(t)= & x+\int_{0}^{t}\left[b_{\varepsilon}\left(\xi_{\varepsilon}(s)\right)+g_{\varepsilon}\left(\xi_{\varepsilon}(s)\right)\right] d s \\
& +\int_{0}^{t} \sigma_{\varepsilon}\left(\xi_{\varepsilon}(s)\right) d w(s), \quad t \in[0, T] .
\end{aligned}
$$

В уравнении $(0.1)\left(w(t), \mathscr{F}_{t}\right)$ - одномерный стандартный винеровский процесс, $\varepsilon$ - малый параметр. Пусть $\lambda, \Lambda$ - постоянные такие, что $0<\lambda \leqslant \Lambda<\infty$. Будем говорить, что пара функций $(f(x), g(x))$ принадлежит классу $\mathscr{L}(\lambda, \Lambda)$, если эти функции измеримы и выполнены неравенства $|f(x)| \leqslant \Lambda, \lambda \leqslant g(x) \leqslant \Lambda$.

Обозначим $a_{\varepsilon}(x)=\sigma_{\varepsilon}^{2}(x)$ и введем условие (I).

У с л о в и е (I). $\mathrm{I}_{1}$. При любом $\varepsilon>0$ функции $b_{\varepsilon}(x), g_{\varepsilon}(x), \sigma_{\varepsilon}(x)$ измеримы, решение уравнения (0.1) единственно в слабом смысле.

$\mathrm{I}_{2}$. Ilapa $\left(g_{\varepsilon}, a_{\varepsilon}\right) \in \mathscr{L}(\lambda, \Lambda)$.

$\mathrm{I}_{3}$. Для любого $x \in E_{1}$

$$
\left|\int_{0}^{x} \frac{b_{\varepsilon}(y)}{a_{\varepsilon}(y)} d y\right| \leqslant \Lambda
$$

Обозначим

$$
\begin{aligned}
& F_{\varepsilon}(x)=\exp \left\{-2 \int_{0}^{x} \frac{b_{\varepsilon}(y)}{a_{\varepsilon}(y)} d y\right\}, \quad f_{\varepsilon}(x)=\int_{0}^{x} F_{\varepsilon}(y) d y, \\
& \beta_{\varepsilon}(x)=2 \int_{0}^{x} F_{\varepsilon}(y)\left[\int_{0}^{y} \frac{b(z)-b_{\varepsilon}(z)}{F_{\varepsilon}(z) a_{\varepsilon}(z)} d z+\beta\right] d y \\
& \alpha_{\varepsilon}(x)=\int_{0}^{x} \frac{a(y)-a_{\varepsilon}(y)\left(1+\dot{\beta}_{\varepsilon}(y)\right)^{2}}{F_{\varepsilon}(y) a_{\varepsilon}(y)} d y \\
& \gamma_{\varepsilon}(x)=\int_{0}^{x} \frac{g(y)-g_{\varepsilon}(y)\left(1+\dot{\beta}_{\varepsilon}(y)\right)}{F_{\varepsilon}(y) a_{\varepsilon}(y)} d y .
\end{aligned}
$$

С введенными функциями свяжем следуюшие условия.

У с л о в и е $(\beta)$. Существуют измеримая ограниченная функция $b(x)$ и постоянная $\beta$ такие, что $\lim _{\varepsilon \rightarrow 0} \beta_{\varepsilon}(x)=0$ для любого $x \in E_{1}$.

У с л о в и е $(\alpha)$. Существует измеримая функция $a(x)$ такая, что $\lambda \leqslant a(x) \leqslant \Lambda$ и для любого $x \in E_{1}$

$$
\lim _{\varepsilon \rightarrow 0} \alpha_{\varepsilon}(x)=0 .
$$


У с л о в и е $(\gamma)$. Существует измеримая ограниченная функция $g(x)$ такая, что для любого $x \in E_{1}$

$$
\lim _{\varepsilon \rightarrow 0} \gamma_{\varepsilon}(x)=0
$$

3 а м е ч а н и е 1. Условиями $(\beta),(\alpha),(\gamma)$ функции $b(x), a(x), g(x)$ и постоянная $\beta$ определяются однозначно (лемма 2 из п. 3 ).

Обозначим через $\left(\mathscr{C}[0, T], \mathscr{C}_{t}\right), t \in[0, T]$, пространство функций $f(t)$, непрерывных на интервале $[0, T], C_{0}^{\infty}\left(E_{1}\right)$ - пространство бесконечно дифференцируемых функций с компактным носителем. Пусть $\mu_{\varepsilon}-$ мера на $\mathscr{C}[0, T]$, соответствуюшая прощессу $\xi_{\varepsilon}$. Будет изучаться слабая сходимость мер $\mu_{\varepsilon}$. Этот тип сходимости обозначим $\Longrightarrow$. Предельная мера $\mu$ будет соответствовать процессу $\xi(t)$ - решению уравнения

$$
\xi(t)=x+\int_{0}^{t} B(\xi(s)) d s+\int_{0}^{t} \sigma(\xi(s)) d w(s) .
$$

Положим $a(x)=\sigma^{2}(x)$ и введем условие (II).

У с л о в и е (II). Пара функций $(B, a) \in \mathscr{L}(\lambda, \Lambda)$.

Сформулируем основные результаты работы

Теорема 1. Справедливы следующие утверждения.

1. Пусть выполнены условия (I), $(\beta),(\alpha) u(\gamma)$. Тогда $\mu_{\varepsilon} \Rightarrow \mu u$ прочесс $\xi(t)$ является решением уравнения $(0.2)$ с бункчией $B(x)=$ $b(x)+g(x)$.

2. Пусть $\mu_{\varepsilon} \Longrightarrow \mu$, выполнень условия (I), (II) и существует измеримая ограниченная функиия $G(x)$ такая, что при каждом $x \in E_{1}$

$$
\lim _{\varepsilon \rightarrow 0} \int_{0}^{x} \frac{g_{\varepsilon}(y)}{a_{\varepsilon}(y)} d y=\int_{0}^{x} G(y) d y .
$$

Тогда выполнено условие $(\beta)$ с функциеи $b(x)=B(x)-a(x) G(x)$ и некоторой постоянной $\beta$, выполнено условие $(\alpha)$ и выполнено условие $(\gamma) c$ бункцией $g(x)=a(x) G(x)$.

3. Пусть $\mu_{\varepsilon} \Longrightarrow \mu$, выполнены условия (I), (II) и существует измеримая ограниченная функция $V(x)$ такая, что для любого $x \in E_{1}$

$$
\lim _{\varepsilon \rightarrow 0} \int_{0}^{x} \frac{b_{\varepsilon}(y)}{a_{\varepsilon}(y)} d y=\int_{0}^{x} V(y) d y .
$$

Тогда выполнено условие $(\beta)$ с функцией $b(x)=a(x) V(x)$ и некоторой постоянной $\beta$, выполнено условие $(\alpha)$, выполнено условие $(\gamma)$ с функии$e \breve{u} g(x)=B(x)-a(x) V(x)$.

Следствие 1. Пусть в уравнении (0.1) функиия $g_{\varepsilon}(x) \equiv 0$. Для сходимости $\mu_{\varepsilon} \Rightarrow \mu$ необходимо и достаточно, чтобы быполнялись условие $(\beta)$ с некоторой постоянной $\beta$ и функцией $b(x)=B(x)$ и условиe $(\alpha)$. 
Следствие 2. Пусть в уравнении (0.1) функиия $b_{\varepsilon}(x) \equiv 0$. Для сходимости $\mu_{\varepsilon} \Longrightarrow \mu$, необходимо и достаточно, чтобы выполнялись условие $(\alpha)$ и условие $(\gamma)$ с фуикиией $g(x)=B(x)$ (в этом случае $\beta=0$, $b(x) \equiv 0)$.

3 а м е ч а н и е 2. Ниже будет доказано, что при условиях пाг. 2 и 3 теоремы 1 существует дифференцируемая функция $f(x)$ такая, что при каждом $x \in E_{1}$

$$
\lim _{\varepsilon \rightarrow 0} f_{\varepsilon}(x)=f(x) .
$$

ІІостоянная $\beta$ из условия $(\beta)$ определяется равенством $(2 \beta+1) \dot{f}(0)=1$.

1. Достаточные условия сходимости. В этом пункте доказывается утверждение 1 сформулированной выше теоремы 1 . Разобьем доказательство на несколько этапов.

a) Докажем слабую компактность семейства мер $\mu_{\varepsilon}$ на пространстве $\mathscr{C}[0, T]$. Применим формулу Ито к функщии $\beta_{\varepsilon}(x)$ :

$$
\begin{aligned}
\beta_{\varepsilon}\left(\xi_{\varepsilon}(t)\right)= & \beta_{\varepsilon}(x)+\int_{0}^{t}\left[b\left(\xi_{\varepsilon}(s)\right)-b_{\varepsilon}\left(\xi_{\varepsilon}(s)\right)+\dot{\beta}_{\varepsilon}\left(\xi_{\varepsilon}(s)\right) g_{\varepsilon}\left(\xi_{\varepsilon}(s)\right)\right] d s \\
& +\int_{0}^{t} \dot{\beta}_{\varepsilon}\left(\xi_{\varepsilon}(s)\right) \sigma_{\varepsilon}\left(\xi_{\varepsilon}(s)\right) d w(s) .
\end{aligned}
$$

Прибавляя это соотношение к уравнению (1), получим

$$
\begin{aligned}
\xi_{\varepsilon}(t)+\beta_{\varepsilon}\left(\xi_{\varepsilon}(t)\right)= & x+\beta_{\varepsilon}(x)+\int_{0}^{t}\left[b\left(\xi_{\varepsilon}(s)\right)+\dot{\beta}_{\varepsilon}\left(\xi_{\varepsilon}(s)\right) g_{\varepsilon}\left(\xi_{\varepsilon}(s)\right)\right] d s \\
& +\int_{0}^{t}\left[1+\dot{\beta}_{\varepsilon}\left(\xi_{\varepsilon}(s)\right)\right]\left(\sigma_{\varepsilon}(s)\right) d w(s) .
\end{aligned}
$$

Обозначим $\eta_{\varepsilon}(t)=\xi_{\varepsilon}(t)+\beta_{\varepsilon}\left(\xi_{\varepsilon}(t)\right)$. Из отмеченных в лемме 3 (см. п. 3 ) свойств функций $\beta_{\varepsilon}(x)$ вытекает, что существует постоянная $K$ такая, ч.то

$$
\left|b(x)+\dot{\beta}_{\varepsilon}(x) g_{\varepsilon}(x)\right|+\left|\sigma_{\varepsilon}(x)\left(1+\dot{\beta}_{\varepsilon}(x)\right)\right| \leqslant K(1+|x|) .
$$

Используя эти оценки, оценки для процесса $\xi_{\varepsilon}(t)$ леммы 5 , из (1.1) стандартно получаем $[3$, с. 120$]$, что

$$
\sup _{t \in[0, T]} \mathbf{E}\left|\eta_{\varepsilon}(t)\right|^{2} \leqslant K\left(1+|x|^{2}\right), \quad \mathbf{E}\left|\eta_{\varepsilon}(t)-\eta_{\varepsilon}(s)\right| \leqslant|t-s|^{2} .
$$

Поэтому $[2$, лемма 2 , с. 355$]$ семейство мер, порожденное процессами $\eta_{\varepsilon}$, слабо компактно на пространстве $\mathscr{C}[0, T]$. В силу леммы 6

$$
\lim _{\varepsilon \rightarrow 0} \mathbf{E} \sup _{t \in[0, T]}\left|\beta_{\varepsilon}\left(\xi_{\varepsilon}(t)\right)\right|^{2}=0 .
$$

Тогда семейство мер, порожденных процессами $\eta_{\varepsilon}(t)-\beta_{\varepsilon}\left(\xi_{\varepsilon}(t)\right)=\xi_{\varepsilon}(t)$, на $\mathscr{C}[0, T]$ также слабо компактно. 
b) Пусть $\Phi(x) \in C_{0}^{\infty}\left(E_{1}\right), \phi_{s}(x)$ - непрерывный ограниченный $C_{s}$-измеримый функционал. Применяя формулу Ито к процессу $\eta_{\varepsilon}(t)$ из (1.1), имеем

$$
\begin{aligned}
\mathbf{E} \phi_{s}\left(\xi_{\varepsilon}\right)[ & \Phi\left(\eta_{\varepsilon}(t)\right)-\Phi\left(\eta_{\varepsilon}(s)\right) \\
-\int_{s}^{t}\{ & \dot{\Phi}\left(\eta_{\varepsilon}(v)\right)\left(b\left(\xi_{\varepsilon}(v)\right)+\dot{\beta}_{\varepsilon}\left(\xi_{\varepsilon}(v)\right) \dot{g}_{\varepsilon}\left(\xi_{\varepsilon}(v)\right)\right) \\
& \left.\left.+\frac{1}{2} \ddot{\Phi}(\hat{\varepsilon}(v)) a_{\varepsilon}\left(\xi_{\varepsilon}(v)\right)\left(1+\dot{\beta}_{\varepsilon}\left(\xi_{\varepsilon}(v)\right)\right)^{2}\right\} d v\right]=0 .
\end{aligned}
$$

Перепишгем это соотношение следующим образом:

$$
\begin{aligned}
\mathrm{E} \phi_{s}\left(\xi_{\varepsilon}\right)[ & \Phi\left(\xi_{\varepsilon}(t)\right)-\Phi\left(\xi_{\varepsilon}(s)\right) \\
& \left.-\int_{s}^{t}\left\{\dot{\Phi}\left(\xi_{\varepsilon}(v)\right)\left[b\left(\xi_{\varepsilon}(v)\right)+g\left(\xi_{\varepsilon}(v)\right)\right]+\frac{1}{2} \ddot{\Phi}\left(\xi_{\varepsilon}(v)\right) a\left(\xi_{\varepsilon}(v)\right)\right\} d v\right] \\
= & I_{\varepsilon}^{1}+I_{\varepsilon}^{2}+I_{\varepsilon}^{3}+I_{\varepsilon}^{4} .
\end{aligned}
$$

В равенстве (1.3)

$$
\begin{aligned}
& I_{\varepsilon}^{1}= \mathbf{E} \phi_{s}\left(\xi_{\varepsilon}\right)\left[\Phi\left(\xi_{\varepsilon}(t)\right)-\Phi\left(\eta_{\varepsilon}(t)\right)-\Phi\left(\xi_{\varepsilon}(s)\right)+\Phi\left(\eta_{\varepsilon}(s)\right)\right] \\
& I_{\varepsilon}^{2}=\mathbf{E} \phi_{s}\left(\xi_{\varepsilon}\right) \int_{s}^{t} {\left[\left(\dot{\Phi}\left(\eta_{\varepsilon}(v)\right)-\dot{\Phi}\left(\xi_{\varepsilon}(v)\right)\right)\left(b\left(\xi_{\varepsilon}(v)\right)+\dot{\beta}_{\varepsilon}\left(\xi_{\varepsilon}(v)\right) g_{\varepsilon}\left(\xi_{\varepsilon}(v)\right)\right)\right.} \\
&\left.+\frac{1}{2}\left(\ddot{\Phi}\left(\eta_{\varepsilon}(v)\right)-\ddot{\Phi}\left(\xi_{\varepsilon}(v)\right)\right) a_{\varepsilon}\left(\xi_{\varepsilon}(v)\right)\left(1+\dot{\beta}_{\varepsilon}\left(\xi_{\varepsilon}(v)\right)\right)^{2}\right] d v \\
& I_{\varepsilon}^{3}= \mathbf{E} \phi_{s}\left(\xi_{\varepsilon}\right) \int_{s}^{t} \dot{\Phi}\left(\xi_{\varepsilon}(v)\right)\left[\left(1+\dot{\beta}_{\varepsilon}\left(\xi_{\varepsilon}(v)\right)\right) g_{\varepsilon}\left(\xi_{\varepsilon}(v)\right)-g\left(\xi_{\varepsilon}(v)\right)\right] d v \\
& I_{\varepsilon}^{4}=\frac{1}{2} \mathbf{E} \phi_{s}\left(\xi_{\varepsilon}\right) \int_{s}^{t} \ddot{\Phi}\left(\xi_{\varepsilon}(v)\right)\left[a_{\varepsilon}\left(\xi_{\varepsilon}(v)\right)\left(1+\dot{\beta}_{\varepsilon}\left(\xi_{\varepsilon}(v)\right)\right)^{2}-a\left(\xi_{\varepsilon}(v)\right)\right] d v
\end{aligned}
$$

c) Покажем, что предел правой части в (1.3) равен нулю. В силу $(1.2)$ и оценок леммы 5 , имеем

$$
\lim _{\varepsilon \rightarrow 0} I_{\varepsilon}^{1}=\lim _{\varepsilon \rightarrow 0} I_{\varepsilon}^{2}=0
$$

Докажем, что

$$
\lim _{\varepsilon \rightarrow 0} I_{\varepsilon}^{3}=0
$$

Для этого в силу леммы 8 достаточно показать, что

$$
\lim _{\varepsilon \rightarrow 0} \mathbf{E}\left|\int_{s}^{t}\left[g_{\varepsilon}\left(\xi_{\varepsilon}(v)\right)\left(1+\dot{\beta}_{\varepsilon}\left(\xi_{\varepsilon}(v)\right)\right)-g(\xi(v))\right] d v\right|=0
$$

Обозначим

$$
\lambda_{\varepsilon}(x)=2 \int_{0}^{x} F_{\varepsilon}(y) \gamma_{\varepsilon}(y) d y
$$


Очевидно, что с некоторой постоянной $K$ справедливы следующие неравенства:

$$
\left|\lambda_{\varepsilon}(x)\right| \leqslant K\left(1+|x|^{3}\right), \quad\left|\dot{\lambda}_{\varepsilon}(x)\right| \leqslant K\left(1+|x|^{2}\right),
$$

и для любого $N<\infty$

$$
\lim _{\varepsilon \rightarrow 0} \sup _{|x| \leqslant N}\left|\lambda_{\varepsilon}(x)\right|=\lim _{\varepsilon \rightarrow 0} \sup _{|x| \leqslant N}\left|\dot{\lambda}_{\varepsilon}(x)\right|=0 .
$$

Применяя формулу Ито, получим

$$
\begin{gathered}
\int_{s}^{t}\left[g_{\varepsilon}\left(\xi_{\varepsilon}(v)\right)\left(1+\dot{\beta}_{\varepsilon}\left(\xi_{\varepsilon}(v)\right)\right)-g\left(\xi_{\varepsilon}(v)\right)\right] d v=\lambda_{\varepsilon}\left(\xi_{\varepsilon}(s)\right)-\lambda_{\varepsilon}\left(\xi_{\varepsilon}(t)\right) \\
\quad+\int_{s}^{t} \dot{\lambda}_{\varepsilon}\left(\xi_{\varepsilon}(v)\right) g_{\varepsilon}\left(\xi_{\varepsilon}(v)\right) d v+\int_{s}^{t} \dot{\lambda}_{\varepsilon}\left(\xi_{\varepsilon}(v)\right) \sigma_{\varepsilon}\left(\xi_{\varepsilon}(v)\right) d w
\end{gathered}
$$

Оценим слагаемые в правой части (1.9). Используя (1.7), имеем

$$
\begin{aligned}
& \mathbf{E} \sup _{t \in[0, T]}\left|\lambda_{\varepsilon}\left(\xi_{\varepsilon}(t)\right)\right| \leqslant \mathbf{E} \sup _{t \in[0, T]}\left|\lambda_{\varepsilon}\left(\xi_{\varepsilon}(t)\right)\right| \chi\left(\sup _{t \in[0, T]}\left|\xi_{\varepsilon}(t)\right| \leqslant K\right) \\
& \quad+\mathbf{E} \sup _{t \in[0, T]}\left|\lambda_{\varepsilon}\left(\xi_{\varepsilon}(t)\right)\right| \chi\left(\sup _{t \in[0, T]}\left|\xi_{\varepsilon}(t)\right|>K\right) \leqslant \mathbf{E} \sup _{t \in[0, T]}\left|\lambda_{\varepsilon}\left(\xi_{\varepsilon}(t)\right)\right| \\
& \quad \times \chi\left(\sup _{t \in[0, T]}\left|\xi_{\varepsilon}(t)\right| \leqslant K\right)+\frac{1}{K}\left(1+\mathbf{E} \sup _{t \in[0, T]}\left|\xi_{\varepsilon}(t)\right|^{6}\right)^{1 / 2} \mathbf{E} \sup _{t \in[0, T]}\left|\xi_{\varepsilon}(t)\right| .
\end{aligned}
$$

Используя (1.8) и оценки леммы 5 , перейдем к пределу в этом неравенстве вначале при $\varepsilon \rightarrow 0$, а затем при $K \rightarrow \infty$. Получим

$$
\lim _{\varepsilon \rightarrow 0} \mathbf{E} \sup _{t \in[0, T]}\left|\lambda_{\varepsilon}\left(\xi_{\varepsilon}(t)\right)\right|=0 .
$$

Аналогично показывается, что

$$
\lim _{\varepsilon \rightarrow 0} \mathbf{E} \sup _{t \in[0, T]}\left|\dot{\lambda}_{\varepsilon}\left(\xi_{\varepsilon}(t)\right)\right|^{2}=0 .
$$

Из соотношений (1.9)-(1.11) легко следует (1.6), а значит, и (1.5).

Аналогично доказывается равенство

$$
\lim _{\varepsilon \rightarrow 0} I_{\varepsilon}^{4}=0 .
$$

Для этого необходимо применить формулу Ито к функции $\hat{\lambda}_{\varepsilon}(x)=$ $2 \int_{0}^{x} F_{\varepsilon}(y) \alpha_{\varepsilon}(y) d y$.

d) Из (1.3), используя соотношения (1.4), (1.5) и (1.12), получим

$$
\begin{aligned}
\lim _{\varepsilon \rightarrow 0} \mathbf{E} \phi_{s}\left(\xi_{\varepsilon}\right)\left[\Phi\left(\xi_{\varepsilon}(t)\right)-\Phi\left(\xi_{\varepsilon}(s)\right)\right. \\
-\int_{s}^{t}\left\{\dot{\Phi}\left(\xi_{\varepsilon}(v)\right)\left[b\left(\xi_{\varepsilon}(v)\right)+g\left(\xi_{\varepsilon}(v)\right)\right]\right. \\
\left.\left.\quad+\frac{1}{2} \ddot{\Phi}\left(\xi_{\varepsilon}(v)\right) a\left(\xi_{\varepsilon}(v)\right)\right\} d v\right]=0
\end{aligned}
$$


Обозначим через $\mu$ одну из предельных точек последовательности $\mu_{\varepsilon}$ и через $E^{\mu}$ - усреднение по мере $\mu$. Если бы функции $b(x), g(x), a(x)$ были непрерывны, то обоснование предельного перехода в (1.13) не вызывало бы трудностей. Для лишь измеримых функций предельный переход обосновывается как в [3, с. 127], с использованием оценки Крылова (лемма 7). Таким образом, из (1.13) имеем

$$
\begin{aligned}
\mathbf{E}^{\mu} \phi_{s}(x)[ & {[\Phi(x(t))-\Phi(x(s))} \\
& \left.-\int_{s}^{t}\left\{\dot{\Phi}(x(v))[b(x(v))+g(x(v))]+\frac{1}{2} \ddot{\Phi}(x(v)) a(x(v))\right\} d v\right]=0 .
\end{aligned}
$$

То есть мера $\mu$ есть решение проблемы мартингалов для коэффициента сноса, равного $b(x)+g(x)$, и коэффициента диффузии, равного $a(x)$. Согласно [1], существует ецинственное решение проблемы мартингалов для указанных коэффициентов. Следовательно, $\mu_{\varepsilon} \Rightarrow \mu$ и мера $\mu$ соответствует решению стохастического уравнения $(0.2)$ с функциями $B(x)=b(x)+g(x), \sigma(x)=\sqrt{a(x)}$. Утверждение 1 теоремы доказано.

2. Необходимые условия сходимости. В этом пункте доказываются необходимые условия сходимости решений стохастических уравнений, сформулированные в теореме 1.

Пусть $h(x)$ - некоторая функщия. Обозначим

$$
M(x)=\exp \left\{-2 \int_{0}^{x} \frac{h(y)}{a(y)} d y\right\}
$$

и введем условие (III).

У с л о в и е (III). Существует постоянная $k$ такая, что пля любого $x \in E_{1}$ :

$$
\begin{aligned}
&\left(\mathrm{III}_{1}\right) \quad \lim _{\varepsilon \rightarrow 0} \int_{0}^{x} F_{\varepsilon}(y) d y=k \int_{0}^{x} M(y) d y, \\
&\left(\mathrm{III}_{2}\right) \lim _{\varepsilon \rightarrow 0} \int_{0}^{x} \frac{d y}{F_{\varepsilon}(y) a_{\varepsilon}(y)}=\frac{1}{k} \int_{0}^{x} \frac{d y}{M(y) a(y)} .
\end{aligned}
$$

Теорема 2. Пусть выполнены условия (I), (III) и пара $(h, a) \in$ $\mathscr{L}(\lambda, \Lambda)$. Пусть, кроме того, существует измеримая ограниченная функиия $G(x)$ такая, что для каждого $x \in E_{1}$

$$
\lim _{\varepsilon \rightarrow 0} \int_{0}^{x} \frac{g_{\varepsilon}(y)}{a_{\varepsilon}(y)} d y=\int_{0}^{x} G(y) d y .
$$

Справедливы следующие утверждения:

1) выполнено условие $(\beta)$ с функцией $b(x)=h(x)$ и постоянной $\beta$ mакой, что $(2 \beta+1) k=1$;

2) выполнено условие $(\alpha)$;

3) выполжено условие $(\gamma)$ с фунхчией $g(x)=a(x) G(x)$. 
Д о к а з а т е л в с т в о. Докажем утверждение 1). Используя условие (III), находим

$$
\begin{aligned}
\lim _{\varepsilon \rightarrow 0} \beta_{\varepsilon}(x) & =\lim _{\varepsilon \rightarrow 0}\left[2 \int_{0}^{x} F_{\varepsilon}(y) \int_{0}^{y} \frac{h(z)}{F_{\varepsilon}(z) a_{\varepsilon}(z)} d z d y+(2 \beta+1) \int_{0}^{x} F_{\varepsilon}(y) d y-x\right] \\
& =2 \int_{0}^{x} M(y) \int_{0}^{y} \frac{h(z)}{M(z) a(z)} d z d y+(2 \beta+1) \int_{0}^{x} M(y) d y-x \\
& =[(2 \beta+1) k-1] \int_{0}^{x} M(y) d y=0 .
\end{aligned}
$$

Условие $(\beta)$ выполнено. Проверим условие $(\alpha)$. Имеем:

$$
\begin{aligned}
\lim _{\varepsilon \rightarrow 0} \alpha_{\varepsilon}(x)=\lim _{\varepsilon \rightarrow 0}\left[\int_{0}^{x} \frac{a(y)}{F_{\varepsilon}(y) a_{\varepsilon}(y)} d y\right. \\
\left.\quad-\int_{0}^{x} F_{\varepsilon}(y)\left(2 \int_{0}^{y} \frac{h(z)}{F_{\varepsilon}(z) a_{\varepsilon}(z)} d z+\frac{1}{k}\right)^{2} d y\right] \\
=\frac{1}{k} \int_{0}^{x} \frac{d y}{M(y)}-k \int_{0}^{x} M(y)\left(\frac{1}{k}\left(\frac{1}{M(y)}-1\right)+\frac{1}{k}\right)^{2} d y=0 .
\end{aligned}
$$

Таким образом, утверждение 2) справедливо. Проверим условие $(\gamma)$ :

$$
\begin{aligned}
\lim _{\varepsilon \rightarrow 0} \gamma_{\varepsilon}(x) & =\lim _{\varepsilon \rightarrow 0}\left[\int_{0}^{x} \frac{a(y) G(y)}{F_{\varepsilon}(y) a_{\varepsilon}(y)} d y-\frac{g_{\varepsilon}(y)}{a_{\varepsilon}(y)}\left(2 \int_{0}^{y} \frac{h(z)}{F_{\varepsilon}(z) a_{\varepsilon}(z)} d z+\frac{1}{k}\right) d y\right] \\
& =\frac{1}{k} \int_{0}^{x} \frac{G(y)}{M(y)} d y-\int_{0}^{x}\left[\frac{2}{k} \int_{0}^{y} \frac{h(z)}{M(z) a(z)} d z+\frac{1}{k}\right] G(y) d y=0 .
\end{aligned}
$$

Теорема полностью доказана.

Положим

$$
\begin{aligned}
& F(x)=\exp \left\{-2 \int_{0}^{x} \frac{B(y)}{a(y)} d y\right\} \\
& Q(x)=\exp \left\{-2 \int_{0}^{x}\left(\frac{B(y)}{a(y)}-G(y)\right) d y\right\} .
\end{aligned}
$$

Д ок а 3 а т е л ь с т в о утверждения 2 теоремы 1 . Обозначим

$$
Z_{\varepsilon}(x)=\exp \left\{-2 \int_{0}^{x} \frac{b_{\varepsilon}(y)+g_{\varepsilon}(y)}{a_{\varepsilon}(y)} d y\right\}, \quad z_{\varepsilon}(x)=\int_{0}^{x} Z_{\varepsilon}(y) d y .
$$

Согласно лемме 10, (п. 3) существует подпоследовательность этих функций (выделим ее индексом $\hat{\varepsilon}$ ) и постоянная $k$ такие, что

$$
\begin{aligned}
\lim _{\hat{\varepsilon} \rightarrow 0} \int_{0}^{x} F_{\hat{\varepsilon}}(y) d y & =\lim _{\hat{\varepsilon} \rightarrow 0} \int_{0}^{x} Z_{\hat{\varepsilon}}(y) \exp \left\{2 \int_{0}^{y} \frac{g_{\hat{\varepsilon}}(z)}{a_{\hat{\varepsilon}}(z)} d z\right\} d y \\
& =k \int_{0}^{x} \exp \left\{-2 \int_{0}^{y} \frac{B(z)-a(z) G(z)}{a(z)} d z\right\} d y \\
& =k \int_{0}^{x} Q(y) d y .
\end{aligned}
$$


Вновь используя лемму 10, получим

$$
\begin{aligned}
\lim _{\hat{\varepsilon} \rightarrow 0} \int_{0}^{x} \frac{d y}{F_{\varepsilon}(y) a_{\varepsilon}(y)} & =\lim _{\hat{\varepsilon} \rightarrow 0} \int_{0}^{x} \frac{1}{a_{\hat{\varepsilon}}(y) Z_{\hat{\varepsilon}}(y)} \exp \left\{-2 \int_{0}^{y} \frac{g_{\hat{\varepsilon}}(z)}{a_{\hat{\varepsilon}}(z)} d z\right\} d y= \\
& =\frac{1}{k} \int_{0}^{x} \frac{1}{a(y)} \exp \left\{2 \int_{0}^{y} \frac{B(z)-G(z) a(z)}{a(z)} d z\right\} d y \\
& =\frac{1}{k} \int_{0}^{x} \frac{d y}{a(y) Q(y)} .
\end{aligned}
$$

Докажем, что постоянная $k$ в (2.1), (2.2) не зависит от выбора указанных подпоследовательностей. Пусть по одной подпоследовательности равенства $(2.1),(2.2)$ выполняются с постоянной $k_{1}$, по другой - с постоянной $k_{2}$. Тогда условие (III) выполнено по одной подпоследовательности с функщией $h(x)=B(x)-a(x) G(x)$ и постоянной $k_{1}$, а по другой подпоследовательности оно выполнено с той же функцией $h(x)$ и постоянной $k_{2}$. В силу теоремы 2 это означает, что условие $(\beta)$ выполняется с функцией $b(x)=B(x)-a(x) G(x)$ и двумя постоянными $\beta_{1}$ и $\beta_{2}$. Согласно лемме 2 , (п. 3) $\beta_{1}=\beta_{2}$, а следовательно, и $k_{1}=k_{2}$. Доказываемое утверждение следует из теоремы 2. Эта часть теоремы 1 доказана.

Д о к а за те л ь с т в у утверждения 3 теоремы 1. Положим

$$
r_{\varepsilon}(x)=\int_{0}^{x} \frac{g_{\varepsilon}(y)}{a_{\varepsilon}(y)} d y
$$

Поскольку $\left|\dot{r}_{\varepsilon}(x)\right| \leqslant \lambda^{-1} \Lambda$, то семейство функций $r_{\varepsilon}(x)$ относительно компактно, и, следовательно, сушествует подпоследовательность $r_{\hat{\varepsilon}}(x)$ и непрерывная функция $r(x)$ такие, что $\lim _{\hat{\varepsilon} \rightarrow 0} r_{\hat{\varepsilon}}(x)=r(x)$. Но тогда

$$
\lim _{\hat{\varepsilon} \rightarrow 0} z_{\hat{\varepsilon}}(x)=\int_{0}^{x} \exp \left\{-2 \int_{0}^{y} V(z) d z-2 r(y)\right\} d y .
$$

С другой стороны, согласно лемме 10 , для некоторой постоянной $k$ справедливо равенство

$$
-2 \int_{0}^{x} V(y) d y-2 r(x)=-2 \int_{0}^{x} \frac{B(y)}{a(y)} d y+\ln k .
$$

Так как $r(0)=0$, то $k=1$ и

$$
r(x)=\int_{0}^{x} \frac{B(y)-V(y) a(y)}{a(y)} d y .
$$

То есть выполнены условия утверждения 2 теоремы 1 с функцией

$$
G(x)=\frac{B(x)-V(x) a(x)}{a(x)} .
$$

Поэтому утверждение 3 следует из утверждения 2. Теорема доказана. 
3. Вспомогательные утверждения. В этом пункте собраны утверждения, использованные выше при доказательстве основных результатов.

Лемма 1. Пусть выполнено условие (I), $l(x)$ - измеримая ограниченная функчия, $\delta$ - постоянная, $u_{\varepsilon}(x)-$ решение уравнения

$$
\begin{gathered}
\frac{1}{2} a_{\varepsilon}(x) \ddot{u}_{\varepsilon}(x)+b_{\varepsilon}(x) \dot{u}_{\varepsilon}(x)=l(x), \\
u_{\varepsilon}(0)=0, \quad \dot{u}_{\varepsilon}(0)=\delta .
\end{gathered}
$$

Eсли $\lim _{\varepsilon \rightarrow 0} u_{\varepsilon}(x)=0$ при каждом $x \in E_{1}$, то $\delta=0$ и почти всюду $l(x)=0$.

Д о к а з а т е л в с т в о. Решение уравнения (3.1) имеет вид:

$$
u_{\varepsilon}(x)=\int_{0}^{x} F_{\varepsilon}(y)\left[2 \int_{0}^{y} \frac{l(z)}{F_{\varepsilon}(z) a_{\varepsilon}(z)} d z+\delta\right] d y .
$$

Обозначим

$$
L_{\varepsilon}(x)=\int_{0}^{x} \frac{d y}{F_{\varepsilon}(y) a_{\varepsilon}(y)} .
$$

При условии (I) имеем с некоторой постоянной $K$ :

$$
\left|f_{\varepsilon}(x)-f_{\varepsilon}(y)\right|+\left|L_{\varepsilon}(x)-L_{\varepsilon}(y)\right| \leqslant K|x-y| .
$$

Следовательно, существуют непрерывные функции $f(x), L(x)$, имеющие почти всюду ограниченные производные $\dot{f}(x), \dot{L}(x)$, а также подпоследовательности $f_{\hat{\varepsilon}}(x), L_{\hat{\varepsilon}}(x)$ такие, что равномерно на компактах

$$
\lim _{\hat{\varepsilon} \rightarrow 0} f_{\hat{\varepsilon}}(x)=f(x)=\int_{0}^{x} \dot{f}(y) d y, \quad \lim _{\hat{\varepsilon} \rightarrow 0} L_{\hat{\varepsilon}}(x)=L(x)=\int_{0}^{x} \dot{L}(y) d y .
$$

Используя (3.3), перейдем в (3.2) к пределу по указанным подпоследовательностям. Получим, что для любого $x \in E_{1}$

$$
\int_{0}^{x} \dot{f}(y)\left[2 \int_{0}^{y} \dot{L}(z) l(z) d z+\delta\right] d y=0 .
$$

Так как функции $f(x)$ и $L(x)$ монотонно возрастают, то $\dot{f}(x)>0$, $\dot{L}(x)>0$. В силу непрерывности функции в квацратных скобках имеем цля любого $y \in E_{1}$ :

$$
2 \int_{0}^{y} \dot{L}(z) l(z) d z+\delta=0 .
$$

Отсюда, положив $y=0$, получим, что $\delta=0$, а затем и $l(x)=0$ почти всюду. Лемма доказана.

Лемма 2. Условиями $(\beta),(\alpha),(\gamma)$ функиии $b(x), a(x), g(x)$ u постоянная $\beta$ определнотся однозначно. 
Д о к а 3 а т е л ь с т в о. Пусть условие $(\beta)$ выполняется для функций $b_{1}(x), b_{2}(x)$ с постоянными $\beta_{1}, \beta_{2}$ соответственно. Положим

$$
\beta_{\varepsilon}^{i}(x)=2 \int_{0}^{x} F_{\varepsilon}(y)\left[\int_{0}^{y} \frac{b_{i}(z)-b_{\varepsilon}(z)}{F_{\varepsilon}(z) a_{\varepsilon}(z)} d z+\beta_{i}\right] d y .
$$

Тогда функция $\beta_{\varepsilon}^{1}(x)-\beta_{\varepsilon}^{2}(x)$ является решением уравнения (3.1) с функцией $l(x)=b_{1}(x)-b_{2}(x)$ и постоянной $\delta=2\left(\beta_{1}-\beta_{2}\right)$. В силу леммы 1 получим, что $\beta_{1}=\beta_{2}$ и почти всюду $b_{1}(x)=b_{2}(x)$.

Пусть условие $(\alpha)$ выполняется с функциями $a_{1}(x)$ и $a_{2}(x)$. Определим функции

$$
\alpha_{\varepsilon}^{i}(x)=\int_{0}^{x} \frac{a_{i}(y)-\alpha_{\varepsilon}(y)\left(1+\dot{\beta}_{\varepsilon}(y)\right)^{2}}{F_{\varepsilon}(y) a_{\varepsilon}(y)} d y .
$$

Из условия $(\alpha)$ и (3.3) получим, что для любого $x \in E_{1}$

$$
\lim _{\hat{\varepsilon} \rightarrow 0}\left(\alpha_{\hat{\varepsilon}}^{1}(x)-\alpha_{\hat{\varepsilon}}^{2}(x)\right)=\int_{0}^{x} \dot{L}(y)\left(a_{1}(y)-a_{2}(y)\right) d y=0 .
$$

Таким образом, $a_{1}(x)=a_{2}(x)$ почти всюду.

Аналогично рассматривается условие $(\gamma)$. Лемма доказана.

Утверждения следующей леммы вытекают из сделанных предположений.

Лемма 3. Пусть выполнены условия (I), $(\beta),(\alpha) u(\gamma)$. Существует постоянная $K$ такая, что справедливы следующие неравенства:

a) $\left|\beta_{\varepsilon}(x)\right| \leqslant K\left(1+|x|^{2}\right),\left|\dot{\beta}_{\varepsilon}(x)\right| \leqslant K(1+|x|)$;

b) $\left|\alpha_{\varepsilon}(x)\right| \leqslant K\left(1+|x|^{3}\right),\left|\dot{\alpha}_{\varepsilon}(x)\right| \leqslant K\left(1+|x|^{2}\right)$;

c) $\left|\gamma_{\varepsilon}(x)\right| \leqslant K\left(1+|x|^{2}\right),\left|\dot{\gamma}_{\varepsilon}(x)\right| \leqslant K(1+|x|)$.

Кроме того, для любого $N<\infty$

d) $\lim _{\varepsilon \rightarrow 0} \sup _{|x| \leqslant N}\left|\beta_{\varepsilon}(x)\right|=0$;

e) $\lim _{\varepsilon \rightarrow 0} \sup _{|x| \leqslant N}\left|\alpha_{\varepsilon}(x)\right|=0$

f) $\lim _{\varepsilon \rightarrow 0} \sup _{|x| \leqslant N}\left|\gamma_{\varepsilon}(x)\right|=0$.

Поскольку функция $f_{\varepsilon}(x)$ при каждом $\varepsilon$ монотонно возрастает, то она имеет обратную функцию, которую обозначим $\phi_{\varepsilon}(x)$.

Лемма 4. При условии (I) справедливы следующие неравенства:

a) $\exp \{-2 \Lambda\} \leqslant F_{\varepsilon}(x) \leqslant \exp \{2 \Lambda\}$

b) $\left|f_{\varepsilon}(x)\right| \leqslant \exp \{2 \Lambda\}|x|$;

c) $\exp \{-2 \Lambda\} \leqslant \dot{\phi}_{\varepsilon}(x) \leqslant \exp \{2 \Lambda\}$;

d) $\left|\phi_{\varepsilon}(x)\right| \leqslant \exp \{2 \Lambda\}|x|$.

Д о к а з а т е л с т в о. Неравенства а), b) есть следствия предположений. Далее,

$$
\dot{\phi}_{\varepsilon}(x)=\frac{1}{\dot{f}_{\varepsilon}\left(\phi_{\varepsilon}(x)\right)}=\frac{1}{F_{\varepsilon}\left(\phi_{\varepsilon}(x)\right)}
$$


Отсюда и из а) следует утверждение с). Очевидно, что $\phi_{\varepsilon}(0)=0$. Используя с), получим $\left|\phi_{\varepsilon}(x)\right|=\left|\phi_{\varepsilon}(x)-\phi_{\varepsilon}(0)\right| \leqslant \exp \{2 L\}|x|$. Лемма доказана.

Лемма 5. Пусть выполнено условие (I). Для любого $m$ существует постоянная $K_{m}$ такая, что

$$
\mathbf{E} \sup _{t \in[0, T]}\left|\xi_{\varepsilon}(s)\right|^{m} \leqslant K_{m}\left(1+|x|^{m}\right) .
$$

Для любых $0 \leqslant s \leqslant t \leqslant T$ существует постоянная $K$ такая, что

$$
\mathbf{E}\left|\xi_{\varepsilon}(t)-\xi_{\varepsilon}(s)\right|^{4} \leqslant K|t-s|^{2} .
$$

Д о к а з а т е л ь с т в о. Применяя формулу Ито, получим

$$
\begin{aligned}
f_{\varepsilon}\left(\xi_{\varepsilon}(t)\right)= & f_{\varepsilon}(x)+\int_{0}^{t} \dot{f}_{\varepsilon}\left(\xi_{\varepsilon}(s)\right) g_{\varepsilon}\left(\xi_{\varepsilon}(s)\right) d s \\
& +\int_{0}^{t} \dot{f}_{\varepsilon}\left(\xi_{\varepsilon}(s)\right) \sigma_{\varepsilon}\left(\xi_{\varepsilon}(s)\right) d w(s) .
\end{aligned}
$$

При сделанных предположениях подынтегральные функции в (3.4) равномерно ограничены. Стандартно $[3$, с. 120$]$ доказываются следующие оценки:

$$
\begin{aligned}
\mathbf{E} \sup _{t \in[0, T]}\left|f_{\varepsilon}\left(\xi_{\varepsilon}(t)\right)\right|^{m} & \leqslant K_{m}\left(1+|x|^{m}\right), \\
\mathbf{E}\left|f_{\varepsilon}\left(\xi_{\varepsilon}(t)\right)-f_{\varepsilon}\left(\xi_{\varepsilon}(s)\right)\right|^{4} & \leqslant K|t-s|^{2} .
\end{aligned}
$$

Используя свойства функций $f_{\varepsilon}(x), \phi_{\varepsilon}(x)$ из леммы 4 , получим

$$
\begin{aligned}
\mathbf{E} \sup _{t \in[0, T]}\left|\xi_{\varepsilon}(t)\right|^{m} & =\mathbf{E} \sup _{t \in[0, T]}\left|\phi_{\varepsilon}\left(f_{\varepsilon}\left(\xi_{\varepsilon}(t)\right)\right)\right|^{m} \\
& \leqslant \exp \{2 m \Lambda\} \mathbf{E} \sup _{t \in[0, T]}\left|f_{\varepsilon}\left(\xi_{\varepsilon}(t)\right)\right|^{m} \leqslant K_{m}\left(1+|x|^{m}\right) .
\end{aligned}
$$

Аналогично доказывается и второе утверждение леммы.

Лемма 6. Пусть выполнены условия (I) $u$ ( $\beta$ ). Тогда

$$
\lim _{\varepsilon \rightarrow 0} \mathbf{E} \sup _{t \in[0, T]}\left|\beta_{\varepsilon}\left(\xi_{\varepsilon}(t)\right)\right|^{2}=0 .
$$

Д ок аз атель с тв о. Очевидно,

$$
\mathrm{E} \sup _{t \in[0, T]}\left|\beta_{\varepsilon}\left(\xi_{\varepsilon}(t)\right)\right|^{2}=I_{1}^{\varepsilon}+I_{2}^{\varepsilon} .
$$

В равенстве (3.5)

$$
\begin{aligned}
& I_{1}^{\varepsilon}=\mathbf{E} \sup _{t \in[0, T]}\left|\beta_{\varepsilon}\left(\xi_{\varepsilon}(t)\right)\right|^{2} \chi\left(\sup _{t \in[0, T]}\left|\xi_{\varepsilon}(t)\right| \leqslant N\right), \\
& I_{2}^{\varepsilon}=\mathbf{E} \sup _{t \in[0, T]}\left|\beta_{\varepsilon}\left(\xi_{\varepsilon}(t)\right)\right|^{2} \chi\left(\sup _{t \in[0, T]}\left|\xi_{\varepsilon}(t)\right| \geqslant N\right) .
\end{aligned}
$$


В силу свойства d) леммы 3

$$
\lim _{\varepsilon \rightarrow 0} I_{1}^{\varepsilon}=0
$$

Далее, учитывая оценки леммы 5, имеем с некоторыми постоянными

$$
\begin{aligned}
I_{2}^{\varepsilon} & \leqslant\left(\mathbf{E} \sup _{t \in[0, T]}\left|\beta_{\varepsilon}\left(\xi_{\varepsilon}(t)\right)\right|^{4}\right)^{1 / 2} \mathbf{P}\left\{\sup _{t \in[0, T]}\left|\xi_{\varepsilon}(t)\right| \geqslant N\right\} \\
& \leqslant \frac{K}{N^{2}}\left(1+\mathbf{E} \sup _{t \in[0, T]}\left|\xi_{\varepsilon}(t)\right|^{8}\right)^{1 / 2} \mathbf{E} \sup _{t \in[0, T]}\left|\xi_{\varepsilon}(t)\right|^{2} \leqslant \frac{K_{m}}{N^{2}} .
\end{aligned}
$$

Переходя к пределу сначала по $\varepsilon \rightarrow 0$, а затем по $N \rightarrow \infty$, получим

$$
\lim _{\varepsilon \rightarrow 0} I_{2}^{\varepsilon}=0 \text {. }
$$

Из (3.5)-(3.7) следует утверждение леммы.

Лемма 7 (оценка Крылова). Пусть выполнены условия (I). Тогда существует постоянная $K_{p}$ такая, ито $\mathbf{E} \int_{0}^{T}\left|\Phi\left(\xi_{\varepsilon}(s)\right)\right| d s \leqslant K_{p}\|\Phi\|_{p}$.

Доказ а те льств о. Прощесс $f_{\varepsilon}\left(\xi_{\varepsilon}(t)\right)$ определяется равенством (3.4). Для него ощенка Крылова справедлива [3, с. 94]: E $\int_{0}^{T}\left|\Psi\left(f\left(\xi_{\varepsilon}(s)\right)\right)\right| d s \leqslant K_{p}\|\Psi\|_{p}$. Используя это неравенство, имеем

$$
\begin{aligned}
\mathbf{E} \int_{0}^{T}\left|\Phi\left(\xi_{\varepsilon}(t)\right)\right| d t & =\mathbf{E} \int_{0}^{T}\left|\Phi\left(\phi_{\varepsilon}\left(f_{\varepsilon}\left(\xi_{\varepsilon}(t)\right)\right)\right)\right| d t \leqslant K_{p}\left[\int_{E_{1}}\left|\Phi\left(\phi_{\varepsilon}(x)\right)\right|^{p} d x\right]^{1 / p} \\
& =K_{p}\left[\int_{E_{1}}|\Phi(y)| F_{\varepsilon}(y) d y\right]^{1 / p} \leqslant K_{p}\|\Phi\|_{p} .
\end{aligned}
$$

Лемма доказана.

Лемма 8. Пусть выполнено условие (I), функция $\psi_{\varepsilon}(t, x)$ такова, что $\operatorname{Esup}_{t \in[0, T]}\left|\psi_{\varepsilon}\left(t, \xi_{\varepsilon}(t)\right)\right|^{2} \leqslant K u \partial л я$ любых $0 \leqslant s \leqslant t \leqslant T$

$$
\lim _{\varepsilon \rightarrow 0} \mathbf{E}\left|\int_{s}^{t} \psi_{\varepsilon}\left(v, \xi_{\varepsilon}(v)\right) d v\right|=0 .
$$

Тогда для любых $\Phi(x) \in C_{0}^{\infty}$ и любых $0 \leqslant s \leqslant t \leqslant T$

$$
\lim _{\varepsilon \rightarrow 0} \mathbf{E}\left|\int_{s}^{t} \Phi\left(\xi_{\varepsilon}(v)\right) \psi_{\varepsilon}\left(v, \xi_{\varepsilon}(v)\right) d v\right|=0
$$

Д ок а з а т е л ь с т в о. Пусть $\left\{t_{i}\right\}$ - некоторое разбиение отрезка $[s, t]: s \leqslant t_{1} \leqslant t_{2} \leqslant \cdots \leqslant t_{n}=t$. Далее,

$$
\begin{aligned}
\mathbf{E}\left|\int_{s}^{t} \Phi\left(\xi_{\varepsilon}(v)\right) \psi_{\varepsilon}\left(v, \xi_{\varepsilon}(v)\right) d v\right| \\
\leqslant \\
\quad+\sum_{i=1}^{n-1}\left|\int_{t_{i}}^{t_{i+1}}\left[\Phi\left(\xi_{\varepsilon}(v)\right)-\Phi\left(\xi_{\varepsilon}\left(t_{i}\right)\right)\right] \psi_{\varepsilon}\left(v, \xi_{\varepsilon}(v)\right) d v\right| \\
\quad+\sum_{i=1}^{n-1} \mathbf{E}\left|\Phi\left(\xi_{\varepsilon}\left(t_{i}\right)\right)\right|\left|\int_{t_{i}}^{t_{i+1}} \psi_{\varepsilon}\left(v, \xi_{\varepsilon}(v)\right) d v\right|
\end{aligned}
$$


ІІри $\varepsilon \rightarrow 0$ второе слагаемое в (3.9) стремится к 0 в силу (3.8). Оценим первое слагаемое в (3.9). Очевидно, что оно не превосходит

$$
\begin{aligned}
& \sum_{i=1}^{n-1}\left(\int_{t_{i}}^{t_{i+1}} \mathbf{E}\left|\Phi\left(\xi_{\varepsilon}(v)\right)-\Phi\left(\xi_{\varepsilon}\left(t_{i}\right)\right)\right|^{4} d v\right)^{1 / 4}\left(\int_{t_{i}}^{t_{i+1}} \mathbf{E}\left|\psi_{\varepsilon}\left(v, \xi_{\varepsilon}(v)\right)\right|^{4 / 3} d v\right)^{3 / 4} \\
& \quad \leqslant K \sum_{i=1}^{n-1}\left(\int_{t_{i}}^{t_{i+1}}\left(v-t_{i}\right)^{2} d v\right)^{1 / 4}\left(t_{i+1}-t_{i}\right)^{3 / 4} \leqslant K \sum_{i=1}^{n-1}\left(t_{i+1}-t_{i}\right)^{3 / 2} \\
& \quad \leqslant K(t-s) \max _{i}\left|t_{i+1}-t_{i}\right|^{1 / 2} .
\end{aligned}
$$

Следовательно, первое слагаемое в (3.9) можно сделать сколь угодно малым равномерно по $\varepsilon$ выбором разбиения. Лемма доказана.

Лемма 9. Пусть $\xi(t)$ есть решение уравнения (0.2), выполнено условие (II) и для некоторых функций $f(x), l(x)$, ююбого $N<\infty u x \in S_{N}$ справедливо равенство

$$
\mathbf{E}_{x}\left[f(\xi(\tau))-\int_{0}^{\tau} l(\xi(s)) d s\right]=f(x),
$$

где $\tau=\inf \{t \geqslant 0:|\xi(t)|=N\}$. Тогда существует постоянная $C$ такая, чmo

$$
f(x)=f(0)+C \int_{0}^{x} F(y) d y+2 \int_{0}^{x} F(y) \int_{0}^{y} \frac{l(z) d z}{F(z) a(z)} d y .
$$

Д о к а з а т е л ь с т в о. Рассмотрим дифференцильное уравнение

$$
\begin{gathered}
\frac{1}{2} a(x) \ddot{u}(x)+B(x) \dot{u}(x)=l(x), \quad-N<x<N, \\
u(-N)=f(-N), \quad u(N)=f(N) .
\end{gathered}
$$

Решение этой задачи имеет вид

$$
u(x)=C_{2}(N)+C_{1}(N) \int_{0}^{x} F(y) d y+2 \int_{0}^{x} F(y) \int_{0}^{y} \frac{l(z) d z}{F(z) a(z)} d y .
$$

Постоянные $C_{1}(N)$ и $C_{2}(N)$ единственным образом определяются из граничных условий. Применим к функции $u(x)$ и процессу $\xi(t)$ формулу Ито:

$$
\mathbf{E}_{x} u(\xi(\tau))=u(x)+\mathbf{E}_{x} \int_{0}^{\tau} l(\xi(s)) d s
$$

Учитывая граничные условия, имеем для любого $x \in S_{N}$

$$
\mathbf{E}_{x}\left[f(\xi(\tau))-\int_{0}^{\tau} l(\xi(s)) d s\right]=u(x) .
$$

Сравнивая (3.10) и (3.11), находим, что $f(x)=u(x)$ для любого $x \in S_{N}$. Отсюда $C_{2}(N)=f(0)$. Очевидно также, что $C_{1}(N)=\dot{u}(0)=\dot{f}(0)=C$. Лемма доказана. 
Обозначим

$$
\begin{aligned}
& Z_{\varepsilon}(x)=\exp \left\{-2 \int_{0}^{x} \frac{b_{\varepsilon}(y)+g_{\varepsilon}(y)}{a_{\varepsilon}(y)} d y\right\} \\
& z_{\varepsilon}(x)=\int_{0}^{x} Z_{\varepsilon}(y) d y, \quad v_{\varepsilon}(x)=\int_{0}^{x} \frac{d y}{a_{\varepsilon}(y) Z_{\varepsilon}(y)} .
\end{aligned}
$$

Лемма 10. Пусть $\mu_{\varepsilon} \Longrightarrow \mu$ и выполнены условия (I), (II). Тогда существуют подпоследовательности $z_{\hat{\varepsilon}}(x), v_{\hat{\varepsilon}}(x)$ и постоянная $k$ такие, что равномерно на компактах

$$
\begin{aligned}
& \lim _{\hat{\varepsilon} \rightarrow 0} z_{\hat{\varepsilon}}(x)=k \int_{0}^{x} F(y) d y, \\
& \lim _{\hat{\varepsilon} \rightarrow 0} v_{\hat{\varepsilon}}(x)=\frac{1}{k} \int_{0}^{x} \frac{d y}{a(y) F(y)} .
\end{aligned}
$$

Д о к а з а т е л с с т в о. При сделанных предположениях для любого $N<\infty$ сушествует постоянная $K_{N}$ такая, что

$$
\sup _{|x| \leqslant N}\left|\dot{z}_{\varepsilon}(x)\right|+\sup _{|x| \leqslant N}\left|\dot{v}_{\varepsilon}(x)\right| \leqslant K_{N} .
$$

То есть семейства функций $z_{\varepsilon}(x), v_{\varepsilon}(x)$ относительно компактны. Следовательно, существуют подпоследовательности $z_{\hat{\varepsilon}}(x), v_{\hat{\varepsilon}}(x)$ и непрерывные функции $z(x), v(x)$ такие, что для любого $N<\infty$

$$
\lim _{\hat{\varepsilon} \rightarrow 0} \sup _{|x| \leqslant N}\left|z_{\hat{\varepsilon}}(x)-z(x)\right|=\lim _{\hat{\varepsilon} \rightarrow 0} \sup _{|x| \leqslant N}\left|v_{\hat{\varepsilon}}(x)-v(x)\right|=0 .
$$

Обозначим $\tau_{\varepsilon}=\inf \left\{t>0,\left|\xi_{\varepsilon}(t)\right|=N\right\}$. Применим формулу Ито:

$$
\mathbf{E}_{x} z_{\varepsilon}\left(\xi_{\varepsilon}\left(\tau_{\varepsilon}\right)\right)=z_{\varepsilon}(x), \quad x \in S_{N} .
$$

Так как $\mu_{\varepsilon} \Longrightarrow \mu$, а функционал $\tau(x(\cdot))=\inf \{t>0:|x(t)|=N\}$ непрерывен на пространстве $\mathscr{C}[0, T]$ по мере $\mu[2$, c. 579], то случайные величины $\xi_{\varepsilon}\left(\tau_{\varepsilon}\right)$ сходятся по распределению к случайной величине $\xi(\tau)$. Далее,

$$
\begin{aligned}
\left|\mathbf{E} z_{\varepsilon}\left(\xi_{\varepsilon}\left(\tau_{\varepsilon}\right)\right)-\mathbf{E} z(\xi(\tau))\right| \leqslant & \mathbf{E}\left|z\left(\xi_{\varepsilon}\left(\tau_{\varepsilon}\right)\right)-z\left(\xi_{\varepsilon}\left(\tau_{\varepsilon}\right)\right)\right| \\
& +\left|\mathbf{E} z\left(\xi_{\varepsilon}\left(\tau_{\varepsilon}\right)\right)-\mathbf{E} z(\xi(\tau))\right| .
\end{aligned}
$$

Перейдем в этом неравенстве к пределу по подпоследовательности $\hat{\varepsilon} \rightarrow 0$. Последнее слагаемое в правой части неравенства сходится к нулю в силу отмеченной выше сходимости $\xi_{\varepsilon}\left(\tau_{\varepsilon}\right)$ к $\xi(\tau)$, а первое слагаемое стремится к нулю в силу (3.14). Имеем,

$$
\mathbf{E}_{x} z(\xi(\tau))=z(x), \quad x \in S_{N}
$$

Так как $z(0)=0$, то в силу леммы 9 существует постоянная $k$ такая, что $z(x)=k \int_{0}^{x} F(y) d y$. Утверждение (3.12) доказано. Докажем (3.13). 
Обозначим $R_{\varepsilon}(x)=\int_{0}^{x} z_{\varepsilon}(y) v_{\varepsilon}(y) d y$. Из (3.12) и (3.14) следует, что существует подпоследовательность $R_{\hat{\varepsilon}}$ такая, что

$$
\lim _{\hat{\varepsilon} \rightarrow 0} R_{\hat{\varepsilon}}(x)=k \int_{0}^{x} F(y) v(y) d y=R(x) .
$$

С другой стороны, применяя формулу Ито, получим $\mathbf{E}_{x}[R(\xi(\tau))-\tau / 2]=$ $R(x), x \in S_{N}$. На основании леммы 9 заключаем, что сушествует постоянная $C$ такая, что функция $R(x)$ допускает представление

$$
R(x)=C \int_{0}^{x} F(y) d y+\int_{0}^{x} F(y) \int_{0}^{y} \frac{d z}{a(z) F(z)} d y .
$$

Сравнивая (3.17) и (3.18), получаем

$$
v(y)=\frac{C}{k}+\frac{1}{k} \int_{0}^{x} \frac{d y}{a(y) F(y)} .
$$

Так как $v(0)=0$, то $C=0$ и равенство (3.13) установлено. Лемма доказана.

4. Примеры. В этом пункте рассматриваются некоторые классы стохастических уравнений и для них на основании полученных выше результатов устанавливаются предельные теоремы. Сохраним введенные выше обозначения.

1. Пусть случайный процесс $\xi_{\varepsilon}(t)$ является решением уравнения

$$
\xi_{\varepsilon}(t)=x+\int_{0}^{t} b_{\varepsilon}\left(\xi_{\varepsilon}(s)\right) d s+\int_{0}^{t} \sigma_{\varepsilon}\left(\xi_{\varepsilon}(s)\right) d w(s),
$$

и предельный процесс $\xi(t)$ есть решение стохастического уравнения

$$
\xi(t)=x+\int_{0}^{t} \sigma(\xi(s)) d w(s) .
$$

Будем считать, что выполнены условия (I) и (II). Для того чтобы для решений уравнений $(4.1),(4.2)$ имела место сходимость $\mu_{\varepsilon} \Longrightarrow \mu$, необходимо и достаточно, чтобы существовала постоянная $k$ такая, что

$$
\lim _{\varepsilon \rightarrow 0} \int_{0}^{x} F_{\varepsilon}(y) d y=k x, \quad \lim _{\varepsilon \rightarrow 0} \int_{0}^{x} \frac{a(y)}{a_{\varepsilon}(y) F_{\varepsilon}(y)} d y=\frac{x}{k} .
$$

Действительно, в этом случае применимо следствие 1. Приведенные условия показывают, что выполнено условие (III) с $F(x)=1$. В силу теоремы 2 выполнено условие $(\beta)$ с функцией $b(x)=0$ и постоянной $\beta$ такой, что $(2 \beta+1) k=1$, выполнено и условие $(\alpha)$.

2. Пусть процесс $\xi_{\varepsilon}(t)$ является решением стохастического уравнения

$$
\xi_{\varepsilon}(t)=x+\frac{1}{\varepsilon} \int_{0}^{t} b\left(\frac{\xi_{\varepsilon}(s)}{\varepsilon}\right) d s+\int_{0}^{t} g\left(\frac{\xi_{\varepsilon}(s)}{\varepsilon}\right) d s+\int_{0}^{t} \sigma\left(\frac{\xi_{\varepsilon}(s)}{\varepsilon}\right) d w(s)
$$


Предположим, что для коэффициентов уравнения (4.3) выполнено условие (I) и существуют следующие пределы:

$$
\begin{aligned}
\lim _{|x| \rightarrow \infty} \frac{1}{x} \int_{0}^{x} \exp \left\{-2 \int_{0}^{y} \frac{b(z)}{a(z)} d z\right\} d y & =B_{1}>0, \\
\lim _{|x| \rightarrow \infty} \frac{1}{x} \int_{0}^{x} \frac{1}{a(y)} \exp \left\{2 \int_{0}^{y} \frac{b(z)}{a(z)} d z\right\} d y & =B_{2}>0, \\
\lim _{|x| \rightarrow \infty} \frac{1}{x} \int_{0}^{x} \frac{g(y)}{a(y)} d y & =B_{3} .
\end{aligned}
$$

В этсм случае условие $(\beta)$ выполнено с постоянной $\beta$ такой, что $2 \beta+1=B_{1}^{-1}$ и функцией $b(x)=0$, условие $(\alpha)$ выполнено с функцией $a(x)=\left(B_{1} B_{2}\right)^{-1}$, условие $(\gamma)$ выполнено с функцией $g(x)=\left(B_{1} B_{2}\right)^{-1} B_{3}$. В силу теоремы 1 предельный процесс для решения уравнения (4.3) есть

$$
\xi(t)=x+\frac{B_{3}}{B_{1} B_{2}} t+\frac{1}{\sqrt{B_{1} B_{2}}} w(t) .
$$

3. Рассмотрим вновь уравнение (4.3), но сделаем другие предположения относительно коэффициентов. Предположим, что

$$
\int_{-\infty}^{\infty}\left|\frac{b(x)}{a(x)}\right| d x<\infty, \quad \int_{-\infty}^{\infty} \frac{b(x)}{a(x)} d x=0
$$

и существуют пределы

$$
\lim _{|x| \rightarrow \infty} \frac{1}{x} \int_{0}^{x} \frac{g(y)}{a(y)} d y=A_{1}, \quad \lim _{|x| \rightarrow \infty} \frac{1}{x} \int_{0}^{x} \frac{d y}{a(y)}=A_{2}>0 .
$$

В этом случае условие $(\beta)$ выполнено с функцией $b(x)=0$ и постоянной $\beta$ такой, что

$$
2 \beta+1=\exp \left\{2 \int_{0}^{\infty} \frac{b(y)}{a(y)} d y\right\} .
$$

Условие $(\alpha)$ выполняется с функцией $a(x)=A_{2}^{-2}$, а условие $(\gamma)$ выполняется с функцией $g(x)=A_{2}^{-1} A_{1}$.

Согласно теореме 1 , предельный процесс в этом случае определяется как

$$
\xi(t)=x+\frac{A_{1}}{A_{2}} t+\frac{1}{A_{2}} w(t) .
$$

4. Пусть случайный прощесс $\xi_{\varepsilon}(t)$ определяется уравнением

$$
\xi_{\varepsilon}(t)=x+\int_{0}^{t}\left[\dot{a}_{\varepsilon}\left(\xi_{\varepsilon}(s)\right)+g_{\varepsilon}\left(\xi_{\varepsilon}(s)\right)\right] d s+\int_{0}^{t} \sigma_{\varepsilon}\left(\xi_{\varepsilon}(s)\right) d w(s) .
$$

Предположим, что

$$
\left(g_{\varepsilon}(x), a_{\varepsilon}(x)\right) \in \mathscr{L}(\lambda, \Lambda) .
$$


Ясно, что условие (I) выполнено. Будем считать, что существуют следуюшие пределы:

$$
\begin{gathered}
\lim _{\varepsilon \rightarrow 0} a_{\varepsilon}(0)=a_{0} \\
\lim _{\varepsilon \rightarrow 0} \int_{0}^{x} \frac{d y}{a_{\varepsilon}(y)}=\int_{0}^{x} A_{1}(y) d y, \quad \lim _{\varepsilon \rightarrow 0} \int_{0}^{x} a_{\varepsilon}(y) d y=\int_{0}^{x} A_{2}(y) d y . \\
\lim _{\varepsilon \rightarrow 0} \int_{0}^{x} \frac{g_{\varepsilon}(x)}{a_{\varepsilon}(y)} d y=\int_{0}^{x} G(y) d y .
\end{gathered}
$$

Пусть, кроме того, $\lambda \leqslant A_{1}(x) A_{2}(x) \leqslant \Lambda$ и $\left|\dot{A}_{1}(x)\right| \leqslant \Lambda$. Простой подсчет показывает, что в этом случае $F_{\varepsilon}(x)=a_{\varepsilon}(0) / a_{\varepsilon}(x)$ и условие $(\beta)$ выполняется с постоянной $\beta$ такой, что $2 \beta+1=\left(a_{0} A_{1}(0)\right)^{-1}$, и функцией

$$
b(x)=-\frac{\dot{A}_{1}(x)}{2 A_{1}^{2}(x) A_{2}(x)} .
$$

Условие $(\alpha)$ выполнено с функцией

$$
a(x)=\frac{1}{A_{1}(x) A_{2}(x)},
$$

а условие $(\gamma)$ выполнено с функцией

$$
g(x)=\frac{G(x)}{A_{1}(x) A_{2}(x)} .
$$

В силу теоремы 1 предельный для решения уравнения (4.4) процесс будет решением уравнения

$$
\xi(t)=x+\frac{1}{2} \int_{0}^{t} \frac{2 G(\xi(s)) A_{1}(\xi(s))-\dot{A}_{1}(\xi(s))}{A_{1}^{2}(\xi(s)) A_{2}(\xi(s))} d s+\int_{0}^{t} \frac{d w(s)}{\sqrt{A_{1}(\xi(s)) A_{2}(\xi(s))}} .
$$

\section{СПИСОК ЛИТЕРАТУРЫ}

1. Веретеяников А.Ю. О сильных решениях стохастических дифференциальных уравнений. - Теория вероятн. и ее примен., 1979, т. XXIV, в. 2, с. 348-360.

2. Гихман И. И., Скороход А.В. Стохастические дифференциальные уравнения и их приложения. Киев: Наукова думка, $1982.612 \mathrm{c.}$

3. Крылов Н. В. Управляемые процессы диффузионного типа. М.: Наука, 1977. $399 \mathrm{c}$.

4. Кулинич Г.Л. О необходимых и достаточных условиях сходимости решений одномерных стохастических диффузионных уравнений при нерегулярной зависимости коэффициентов от параметра. - Теория вероятн. и ее примен., 1982, т. XXVII, в. 4 , с. $795-801$. 